\title{
Morphological Alterations in the Thalamus, Striatum, and Pallidum in Autism Spectrum Disorder
}

\author{
Manuela Schuetze ${ }^{*, 1,2,3}$, Min Tae M Park ${ }^{4,5}$, Ivy YK Cho ${ }^{1,2}$, Frank P MacMaster ${ }^{1,2,6,7,8}$, M Mallar Chakravarty,9 \\ and Signe L Bray ${ }^{1,2,10}$ \\ 'Child and Adolescent Imaging Research (CAIR) Program, University of Calgary, Calgary, AB, Canada; ${ }^{2}$ Alberta Children's Hospital Research \\ Institute, University of Calgary, Calgary, AB, Canada; ${ }^{3}$ Hotchkiss Brain Institute, University of Calgary, Calgary, AB, Canada; ${ }^{4}$ Cerebral Imaging \\ Centre, Douglas Mental Health University Institute, Montreal, QC, Canada; ${ }^{5}$ Schulich School of Medicine and Dentistry, Western University, \\ London, ON, Canada; ${ }^{6}$ Departments of Psychiatry and Pediatrics, University of Calgary, Calgary, AB, Canada; ${ }^{7}$ Mathison Centre for Mental Health \\ Research and Education, Hotchkiss Brain Institute, Calgary, AB, Canada; ${ }^{8}$ Strategic Clinical Network for Addictions and Mental Health, Alberta \\ Health Services, Calgary, AB, Canada; ${ }^{~}$ Departments of Psychiatry and Biomedical Engineering, McGill University, Montreal, QC, Canada; \\ ${ }^{10}$ Departments of Pediatrics and Radiology, University of Calgary, AB, Canada
}

Autism spectrum disorder (ASD) is a common neurodevelopmental disorder with cognitive, motor, and emotional symptoms. The thalamus and basal ganglia form circuits with the cortex supporting all three of these behavioral domains. Abnormalities in the structure of subcortical regions may suggest atypical development of these networks, with implications for understanding the neural basis of ASD symptoms. Findings from previous volumetric studies have been inconsistent. Here, using advanced surface-based methodology, we investigated localized differences in shape and surface area in the basal ganglia and thalamus in ASD, using TI-weighted anatomical images from the Autism Brain Imaging Data Exchange (373 male participants aged 7-35 years with ASD and 384 typically developing). We modeled effects of diagnosis, age, and their interaction on volume, shape, and surface area. In participants with ASD, we found expanded surface area in the right posterior thalamus corresponding to the pulvinar nucleus, and a more concave shape in the left mediodorsal nucleus. The shape of both caudal putamen and pallidum showed a relatively steeper increase in concavity with age in ASD. Within ASD participants, restricted, repetitive behaviors were positively associated with surface area in bilateral globus pallidus. We found no differences in overall volume, suggesting that surface-based approaches have greater sensitivity to detect localized differences in subcortical structure. This work adds to a growing body of literature implicating corticobasal ganglia-thalamic circuits in the pathophysiology of ASD. These circuits subserve a range of cognitive, emotional, and motor functions, and may have a broad role in the complex symptom profile in ASD. Neuropsychopharmacology (2016) 4I, 2627-2637; doi: I0.1038/npp.2016.64; published online 8 June 2016

\section{INTRODUCTION}

Autism spectrum disorder (ASD) is a neurodevelopmental disorder affecting 1 in 68 children (CDC, 2014). In addition to impairment in social communication, ASD diagnostic criteria include restricted, repetitive patterns of interests, behavior, and activities (American Psychiatric Association, 2013). Furthermore, cognitive inflexibility (Geurts et al, 2009) and abnormalities in motivated behaviors (Kohls et al, 2013) have been observed as secondary symptoms. These behavioral domains (motor, cognitive, and emotional) rely on a set of parallel corticostriatal-thalamic circuits (Alexander et al, 1986).

Neurons projecting from the dorsolateral prefrontal cortex to the caudate head are involved in shifting attention and

*Correspondence: M Schuetze, Department of Neuroscience, University of Calgary, clo Glenda Maru, 4th Floor, C4-100-07, Alberta Children's Hospital, 2888 Shaganappi Trail NW, Calgary, AB, Canada T3B 6A8, Tel: + I 403955 2966, Fax: + I 403955 2772,

E-mail: manuschuetze@gmail.com

Received 3 February 2016; revised 18 April 2016; accepted 20 April 2016; accepted article preview online 29 April 2016 strategies when tasks demand change (Bonelli and Cummings, 2007). This skill set is impaired in ASD as 'insistence on sameness' is part of the broader core symptom of restricted, repetitive behaviors (Langen et al, 2014). The orbitofrontal circuit with neurons projecting from medial inferior frontal gyrus to ventromedial caudate and further to dorsal globus pallidus has been described as the 'neocortical representation of the limbic system' (Bonelli and Cummings, 2007). Lesion studies have shown social deficits resulting from injury to these areas-another core symptom in ASD (Bechara, 2000; Hillis, 2014). Furthermore, the caudate nucleus of the dorsal striatum mediates habitual and rigid behaviors, which are related to restricted and repetitive behaviors (Graybiel, 2008). Abnormal connectivity in circuits relayed via the thalamus have also been associated with cognitive impairments in ASD (Nair et al, 2013). Thus, while cortical regions involved in language and social comprehension have been the focus of much ASD research (Nomi and Uddin, 2015), insights into the neural basis of ASD symptoms may also come from studying abnormalities in the basal ganglia and thalamus. 
Structural magnetic resonance imaging (MRI) can be used to measure morphometric properties of subcortical structures. Although this approach cannot pinpoint specific cellular differences, it can provide clues about atypical development of brain regions, and the relation to symptoms. Using this approach, volumetric MRI studies have found overall enlargement of the striatum (Hollander et al, 2005; Langen et al, 2007), pallidum (Giedd et al, 1996), and thalamus (Lin et al, 2015) in participants with ASD compared with typically developing (TD) control participants. However, there are notable inconsistencies in this literature with reports of decreased (McAlonan et al, 2008) or no differences in volume (Haar et al, 2014) in ASD.

A potential weakness of volumetric studies is they often involve averaging volume measurements over a given structure. As the basal ganglia and thalamus are composed of subregions with distinct function and connectivity (Alexander et al, 1986), this risks diluting group differences localized to a particular circuit. A more sensitive measure of structural changes in subcortical regions might be surface modeling. Surface modeling of subcortical structures enables localized measurement of surface area and shape (in terms of concavity and convexity; Chakravarty et al, 2015). This approach has enabled unprecedented detail into sexually dimorphic developmental changes (Raznahan et al, 2014), and localization of abnormalities in brain disorders such as schizophrenia and attention deficit hyperactivity disorder (Chakravarty et al, 2015; Shaw et al, 2014). This work suggests that surface modeling is sensitive to cellular differences in specific subnuclei of the striatum, which can in turn be linked to molecular pathways (Shaw et al, 2014). For example, atypical surface contraction of the ventral striatum, a region associated with reward processing and dopaminergic innervations, was found in children and adolescents with ADHD (Shaw et al, 2014).

Despite 'extreme locus genetic heterogeneity' in ASD (O'Roak et al, 2012) and an estimated several hundred candidate genes (Krumm et al, 2014), converging genetic evidence points to the striatum as a site of abnormalities in ASD (reviewed in Fuccillo, 2016). Indeed, an MR study that applied hierarchical clustering to a set of genetic models of ASD found a cluster with significant regional relative volume differences (both decreased and increased) in 17 different mouse models of ASD (Ellegood et al, 2015). More specifically, Met receptor mutations have been related to ASD (Campbell et al, 2007) and mouse models have shown that Met receptor disruption in the cortex increased total dendritic arbor length and spine volume of striatal medium spiny neurons (Smith et al, 2012). Furthermore, loss-of-function models of contactinassociated protein-like 2 have shown typical ASD behaviors and decreased numbers of interneurons in the striatum (Peñagarikano et al, 2011). Interestingly, these two findings can potentially result in both increased and decreased striatal volume and highlight the benefit of surface modeling as it enables localization of atypical structure with greater precision than volumetric approaches.

In ASD, previous studies have shown atypical connectivity between specific subcortical nuclei and cortical regions (Belmonte et al, 2004), for example, lower connection probability for thalamocortical networks (Nair et al, 2013). Surface modeling may also be sensitive to localized decreases in neuronal density associated with such a connectivity difference. On the other hand, more global mechanisms such as reduced apoptosis in ASD (Wei et al, 2014) or dysregulated axonal growth (McFadden and Minshew, 2013) may lead to broader differences that are detectable in overall volume. To date, only one study has investigated shape differences in ASD, which found decreased reciprocal social and communication skills in ASD participants associated with a more convex shape of the caudate head, and decreased motor skills associated with a more concave shape of bilateral putamen (Qiu et al, 2010).

Taken together, animal models and human neuroimaging provide tantalizing evidence for structural abnormalities that may reflect atypical development of subcortical structures, and associated functional circuits. However, findings have been inconsistent, perhaps because studies have varied in terms of age, medication status, intellectual functioning, and symptom severity of participants, used different methods to calculate volume, and have often assessed relatively small sample sizes. It is also increasingly clear that as the brain undergoes pronounced changes across childhood and adolescence, differences in ASD should be considered in a developmental context (Langen et al, 2014).

The rationale for the present study is that as the basal ganglia and thalamus are central to many behaviors affected in ASD, these regions may undergo atypical development at the cellular level. Surface modeling offers the opportunity to identify localized structural abnormalities that may reflect differences in cellular development; these in turn may impact how these regions form structural connections to the broader networks supporting affected behaviors. In the present study, we compared structure of thalamus, pallidum, and striatum of males with and without ASD between the ages of 7 and 35 years, using data from a large multisite sample (Autism Brain Imaging Data Exchange (ABIDE); Di Martino et al, 2014). By combining volumetric and surface-based analyses, we modeled effects of age, ASD diagnosis and their interaction, to uncover both persistent differences that may emerge early in childhood, and differences in trajectories from childhood into adulthood, both of which may provide insight into the neuroanatomical basis of ASD symptoms. We hypothesized that while volumes would not show group differences (Haar et al, 2014), we would identify localized regions of surface area and shape differences in ASD. As ASD symptoms cut across functional zones of the basal ganglia and thalamus (Alexander et al, 1986), we further hypothesized widespread morphological differences between individuals with ASD and a TD control group.

\section{MATERIALS AND METHODS}

\section{ABIDE Participants and MR Imaging Data}

This study analyzed T1-weighted anatomical images from the ABIDE, which includes 539 individuals with ASD and 573 TD participants (Di Martino et al, 2014), aged 6.47-64 years, collected as part of 20 studies at 17 different sites (MR acquisition protocol details in Supplementary Table S1). All sites collected images using $3 \mathrm{~T}$ MRI systems, with voxel dimensions ranging from 1 to $1.69 \mathrm{~mm}^{3}$. As not all sites collected female participants and sampling was relatively sparse for $<7$ and $>35$ years, we selected male participants between the age of 7 and 35 years. Following quality control, 
described below, our final sample consisted of 373 ASD and 384 TD participants. For ASD participants, diagnosis was confirmed either with the Autism Diagnostic Observation Schedule (ADOS; Lord et al, 1999) or the Autism Diagnostic Interview-Revised (ADI-R; Rutter et al, 2003). Participant demographics are shown in Supplementary Table S2. Most sites (16/17) reported excluding participants with psychiatric or neurological conditions, including, but not limited to, major depression, schizophrenia, epilepsy, and history of traumatic brain injury. Within each sample, TD participants were matched to ASD participants relative to age. In 14 of 17 sites history of current psychoactive medication was collected (see Supplementary Table S2 for details). Participants were asked to withhold any medication use before scan in $5 / 17$ sites.

\section{MAGeT Automated Segmentation Algorithm and Surface-Based Analysis}

We used an automated segmentation algorithm (Multiple Automatically Generated Templates Brain Segmentation, MAGeT Brain; Chakravarty et al, 2013) to extract subcortical volume and shape metrics from T1 images. MAGeT brain uses a 'multiatlas'-based strategy to reduce bias and enhance accuracy of segmentations. To summarize, a ground-truth atlas was manually defined using serial histological data (Chakravarty et al, 2006) and warped to the average of 27 contrast-enhanced MRIs from one individual (Holmes et al, 1998). This atlas was then used to define a set of templates through nonlinear warping to a set of 31 subjects (from both children and adults, mean age: 27.72 years; SD: 13.98 ) chosen from our sample based on the best initial atlas-to-template registrations, from a larger pool of candidate templates ( 250). MTMP manually inspected labels for all 250 candidate templates, excluding suboptimal segmentations from the template library. This resulted in 31 final template library images, which were selected as those with highly accurate segmentations, comparable to manual segmentation. All other subjects were then warped to these 31 subjects, giving 31 candidate segmentations. Final segmentations were decided using a voxel voting procedure, that is, each voxel received the label that occurs most commonly across candidate segmentations (Collins and Pruessner, 2010).

Note that while the mean age of participants included in the template is older than the sample mean, to optimize accuracy of final segmentations, it is a higher priority to ensure accurate initial atlas-to-template registrations by choosing candidates that best match the initial atlas than to find candidates that best represent the sample.

The quality of anatomical images was not assessed before entry in the segmentation pipeline. However, following segmentation, quality was assessed by three authors (MS, MTMP, and IYKC) who visually checked whether edges between subcortical nuclei found by the algorithm and cerebrospinal fluid or white matter tracts correctly matched subcortical borders (similar to manual tracing, which is regarded the gold standard for segmentation). At this stage, images were also assessed for overall quality, and eliminated if poor contrast or 'ringing' were observed. Only if all three authors classified the segmentation of the algorithm as incorrect (eg, because of image distortion due to head motion leading to low contrast between the different tissue types) were the subjects removed from the analysis. We removed 79 subjects (mean age: 14.58 years; SD: 6.9) because of failed segmentation or low image quality. Supplementary Figure S1 shows examples of a successful segmentation (A) and an excluded participant because of low image quality (B). Supplementary Table S3 gives a demographic overview of all excluded participants.

Measures of shape, surface area, and volume for thalamus, striatum, and pallidum were obtained as described previously (Chakravarty et al, 2015; Raznahan et al, 2014). Volumes from segmentations for bilateral thalamus, pallidum, ventral striatum, putamen, and precommissural and postcommissural caudate were analyzed. Shape and surface area analyses used an adaptation of previously described surface-based methodology (Lerch et al, 2008) wherein representations of the surfaces are estimated using the marching cubes algorithm (Lorensen and Cline, 1987) and morphologically smoothed using the AMIRA software package (Visage Imaging, San Diego, CA). This procedure yields 31 possible surface representations per subject that are then merged by creating a new surface representation by estimating the median coordinate representation at each location. To estimate vertex-wise surface area, a third of the surface of each triangle is assigned to each vertex within the triangle. The surface area value stored at each vertex is the sum of all such assignments from all connected triangles. Finally, surface-area values were blurred with a surface-base diffusion-smoothing kernel (5 and $3 \mathrm{~mm}$ for the striatum/ thalamus and pallidum, respectively). To estimate vertexwise shape, the nonlinear portions of the 31 transformations that map each subject to the templates were concatenated and averaged across the template library to limit the effects of noise and error and to increase precision and accuracy (Borghammer et al, 2010; Frey et al, 2011). The dot product between the nonlinear deformation vector (the inverse of the averaged atlas-to-subject transformation) and the surface normal (a unit vector describing the direction perpendicular to the surface) is estimated and provides a local measure of inward or outward displacement along the normal. Note that before estimating the dot product between the normal to the surface and the deformation field, we explicitly model and remove global linear effects not originally accounted for in the initial linear transformations. Intracranial volume included the ventricles and was determined by computing the Jacobian determinant of the linear registration to a common target (Montreal Neurological Institute template13, ICBM 2009c Nonlinear Symmetric template; http://www.bic. mni.mcgill.ca/ServicesAtlases/). In total, 21156 vertices were obtained across all subcortical regions.

\section{Statistical Analysis}

Vertex-wise shape and surface area measures, as well as volume, were analyzed using linear mixed models. To account for the hierarchical nature of data (data were collected across different sites with different acquisition parameters), we included 'site' as a random (grouping) variable in all models. Surface area and volume models included effects of diagnosis, age, a diagnosis-by-age interaction term, full-scale IQ (FIQ), and total intracranial volume. The FIQ term was included in our models to identify differences in subcortical morphology specific to ASD 
diagnosis and not related to differences in IQ. We corrected for intracranial instead of brain volume because traditional brain extraction methods such as BEaST (Eskildsen et al, 2012) show disadvantages for images from multisite databases as parameter tuning and library generation needs to be carried out separately per site. Resulting whole-brain segmentations differ significantly in terms of accuracy. Using intracranial volume inferred from a linear registration gives an unbiased estimate of head size and has been implemented in recent large-scale analyses (http://enigma.ini.usc.edu/). Shape models were similar but did not include intracranial volume as linear effects are explicitly removed from the deformation fields from which these metrics are derived. Similar to Raznahan et al. (2014), linear rather than quadratic or cubic effects of age were modelled because only a small portion of subcortical vertices in our sample showed significant quadratic (shape: $1.23 \%$; surface area: $1.83 \%$ ) or cubic (shape: $3.93 \%$; surface area: $24.5 \%$ ) age effects, while $49.8 \%$ of vertices showed significant linear effects of age for shape and $41.3 \%$ of vertices showed significant linear effects of age for surface area. As $24.5 \%$ of vertices showed cubic age effects on surface area, we also ran a model that included a cubic age term (significant regions shown in Supplementary Figure S2). For volume, we used a step-down regression approach proceeding from a cubic to quadratic and linear model and determined that linear trajectories gave the best fit informed by $P$-values for each model. All analyses were corrected for multiple comparisons using false discovery rate (FDR) thresholded at 5\% (Genovese et al, 2002); $q$-values are reported. Brain-view 2 was used to overlay significant $q$-values on subcortical structures $(q<0.05)$.

As medication has been reported to influence subcortical morphology (Ballmaier et al, 2008), two additional models were run to ensure robustness of our findings. First, we repeated our analysis for the subset of non-medicated participants, which included 239 ASD and 310 TD participants. Second, we ran a model including medications categorized by class as covariates, including participants from the 14/17 sites that collected medication information. This analysis included a sample of 253 ASD and 260 TD participants. Medication was categorized into four groups: stimulants, antidepressants, antipsychotics, and others (eg, anticonvulsants, lithium).

Owing to long-term effects of medication on brain structures (eg, Ho et al, 2011; Navari and Dazzan, 2009), participants who were withholding medication at the time of scan were included as medicated participants. Two sites that did not report medication information were excluded from this analysis (Supplementary Table S2).

To investigate the relationship between symptom severity and subcortical morphology, we conducted a subgroup analysis on 271 ASD participants for whom ADOS scores had been collected. The ADOS assessment differs based on an individual's language and developmental state: Module 4 is used to assess verbally fluent adolescents and adults, Module 3 is used for verbally fluent children aged 12-16 years, Module 2 is used for individuals with some phrase speech and Module 1 is used for younger children or individuals who do not use phrase speech. These differences make a comparison of participants who have been assessed with different modules difficult. Hence, ADOS scores were used to calculate Calibrated Symptom Severity scores (CSS;
Gotham et al, 2009; Hus and Lord, 2014) to better compare participants who were assessed at different ages and with different ADOS modules. Note that CSS scores are derived from ADOS scores and participants without ADOS scores could not be included in this analysis. CSS scores present an overall picture of ASD symptom severity across both domains of social communication and restricted interests and repetitive behaviors. As specific symptoms may be related to differences in the structure of specific striatal subregions, we additionally investigated associations with specific subscales. Caudate and putamen have been implicated in what is called 'insistence on sameness', which belongs to the core symptom of restricted, repetitive behaviors (Langen et al, 2014). We therefore were particularly interested in taking a closer look at the association between the ADOS subscales of stereotypical behavior and subcortical morphology. ASD participants for whom these scores were available (ADOS: $N=223$ ) were included in this analysis.

\section{RESULTS}

\section{Demographics}

Supplementary Table S2 shows demographic data for all participants included in our analysis. Although participants were matched for age in each study contributed to ABIDE, after quality control there was a small (Cohen's $d=0.14$ ) but statistically significant difference in age, with ASD participants on average younger than TD participants $\left(t_{740}=-2.5\right.$, $p<0.05$, ASD range: $7-34.46$, mean: $15.79, \mathrm{SD}: 5.9$, TD range: 7-35, mean: 16.74, SD: 6.46, Supplementary Figure S3A). Ages for each diagnostic group are plotted in Supplementary Figures S3A and B. In a follow-up analysis, we used a reduced sample that did not significantly differ by age $\left(t_{731}=-1.79\right.$, $p>0.5, \mathrm{TD}=375, \mathrm{ASD}=373$ ). Matching of participants was performed for each site individually by step-wise removal of participants from the higher end of the age range until the remaining samples were not significantly different in age. The results were unchanged, and we therefore used the full sample for all analyses presented below. A linear mixed model with data acquisition site as random factor also showed that FIQ was significantly lower $\left(t_{740}=-5.81, p<0.001\right)$ in the ASD group (range: 61-148, mean: 105.24, SD: 16.64) compared with the TD group (range: 73-148, mean: 111.73, SD: 12.22, Supplementary Figures S1C and D). Both age and FIQ were included as covariates in all analyses.

\section{Effects of Age}

To provide context for effects of diagnosis, we began by visualizing the effect of age on shape and surface area across all participants (Figure 1).

Thalamus. Bilateral caudal thalami showed a more concave shape with age, while rostral peaks showed a more convex shape with age. Surface area of bilateral caudal thalami showed a contraction, while lateral thalamus expanded with age.

Pallidum. Rostral pallidum showed a more convex shape with age, while caudal pallidum showed a more concave shape with age. Similarly, surface area expanded in caudal parts of the pallidum, while it contracted in rostral parts. 


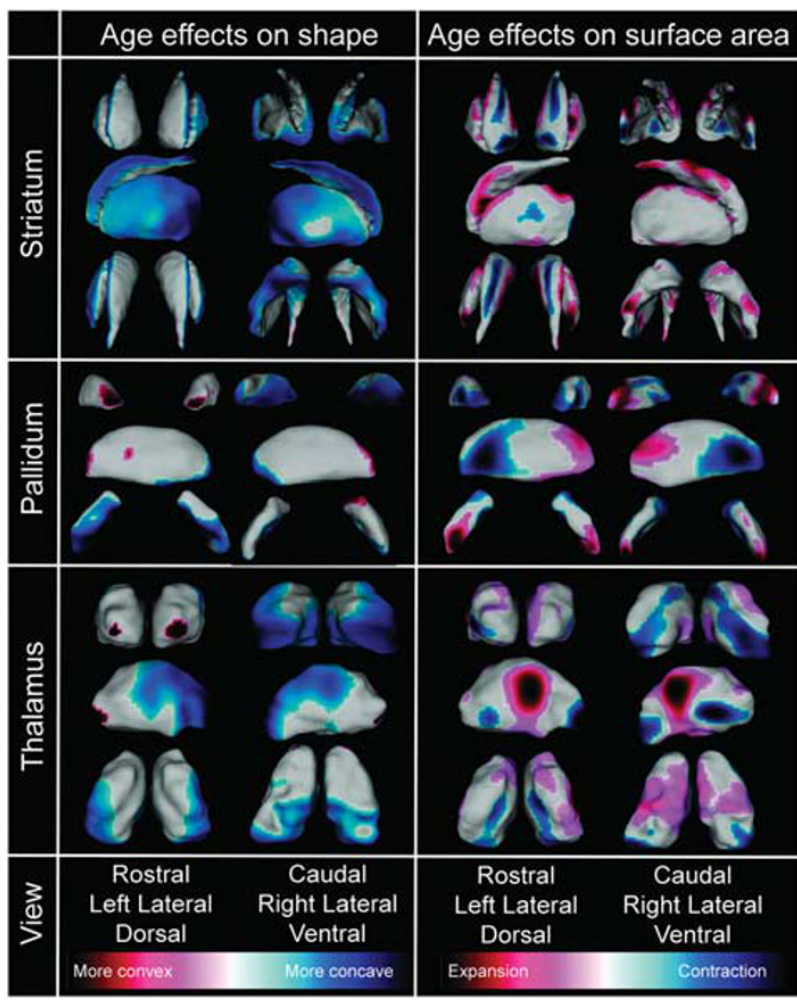

Figure I Effects of age on shape and surface area. Thresholded t-values for the effect of age on shape (left panel) and surface area (right panel) are overlaid on subcortical regions. For shape, cool colors indicate areas that take on a more concave shape over time, and warm colors indicate a more convex shape. For surface area, cool colors indicate areas that contract with increasing age and warm colors indicate an expansion.

Striatum. Lateral striatum showed a more concave shape with age. Surface area of dorsal and rostral striatum showed a contraction with age, while surface area of dorsal putamen and lateral caudate expanded with age.

\section{Effects of Diagnosis}

Thalamus. No significant main effects or interactions with diagnosis were found in thalamus volume. However, for surface area, we found clusters showing a main effect of ASD diagnosis in both the right and left thalamus (right peak $q=0.011$; left peak $q=0.014$ ). For the left, the clusters were in the lateral and medial posterior, as well as medial inferior thalamus (Figure 2a), whereas in the right thalamus the clusters were superior and posterior (Figure 2b). These peak regions showed greater surface area in ASD compared with TD participants (Figure 2, inlay). This interaction effect was not significant when the cubic term was added in the model; however, significant vertices from the cubic model were not overlapping with those for which a main effect of diagnosis was found, suggesting that the cubic model was not appropriate for those vertices where significant diagnosis effects were found. We found a significant effect of diagnosis on shape in the medial right thalamus (peak $q=0.011$; Figure 3), where shape was more concave in the ASD group (Figure 3, inlay). No significant diagnosis-by-age interactions were found for shape or surface area in the thalamus.
Pallidum. We found a significant diagnosis-by-age interaction on shape for the right pallidum (peak $q=0.007$ ), peaking at the posterior aspect (Figure 4a). The shape of the posterior part of the right pallidum became more concave over time in both groups; however, the decline was steeper for ASD participants (Figure 4a, inlay).

Striatum. Only a trend toward larger volume in ASD compared with TD participants was found for the left postcommissural caudate $\left(t_{737}=2.41, p=0.01\right)$ and right pre- and postcommissural caudate $\left(t_{737}=2.08, p=0.03\right.$ and $t_{737}=1.96$, $p=0.0502$ ); these effects did not survive FDR correction. We did not find main effects of diagnosis, or diagnosis-by-age interactions for surface area in the striatum (putamen or caudate), or group differences in shape. However, we did find a significant diagnosis-by-age interaction effect on shape in the right putamen (peak $q=0.018$ ), peaking at the posterior part of the right putamen (Figure $4 \mathrm{~b}$ ). The shape of the posterior right putamen became more concave over time in both groups; however, the decline was steeper in the ASD group (Figure 4b, inlay).

\section{Effects of FIQ}

We found a significant effect of FIQ on surface area in left $(q=0.024)$ and right $(q=0.006)$ thalamus, as well as in left pallidum $(q=0.03)$. Individuals with a higher FIQ showed contracted surface area in left and right thalamus, and expanded surface area in left pallidum (Figure 5a). We also found a significant effect of FIQ on shape in left striatum (Figure 5b; medial aspect in left panel and lateral aspect in right panel). With increasing FIQ we found a more convex shape of the left lateral putamen $(q=0.04)$ and dorsal caudate tail $(q=0.04)$ and a more concave shape of the medial putamen $(q=0.04)$ and medial caudate tail $(q=0.04)$.

\section{Symptom Severity}

Using the CSS scores, we did not find effects of symptom severity for volume, shape, or surface area in any region. However, in a follow-up analysis in the clusters that showed a significant main effect of diagnosis, we found that surface area in the left thalamus was positively modulated by symptom severity (Figure 6).

When examining subscales from the ADOS (social interaction, communication, and restricted/repetitive behaviors or interests), we found greater surface area in bilateral dorsal medial part of the globus pallidus $(q<0.01$; Figure 7) in individuals with more severe restricted, repetitive symptoms on the $\mathrm{ADOS}$ assessment.

\section{Medication}

When analyzing the subsample of non-medicated participants, all shape measures remained significant: main effect of right thalamus $(q=0.009)$, diagnosis-by-age interactions for right putamen $(q=0.01)$, and right pallidum $(q=0.002)$. The main effect of diagnosis remained significant for surface area of the left thalamus $(q=0.03)$, but not for surface area of right thalamus. In a separate model, we included medication as a covariate; here, main effects of diagnosis remained significant for surface area of left and right thalamus $(q=0.03$ and 0.02 , respectively), but not for the shape of 

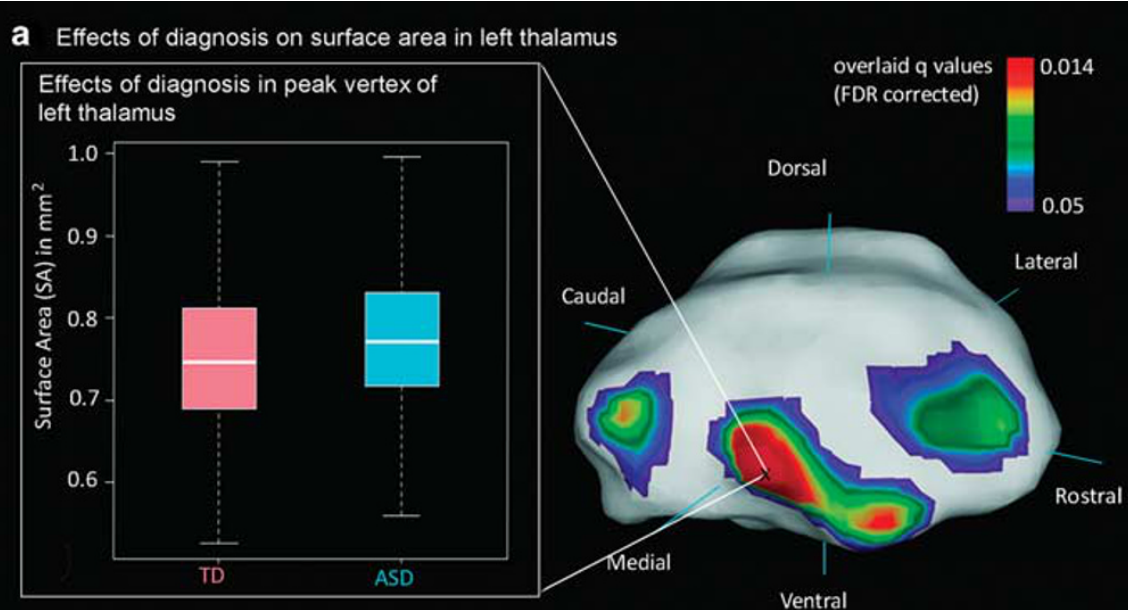

b Effects of diagnosis on surface area in right thalamus

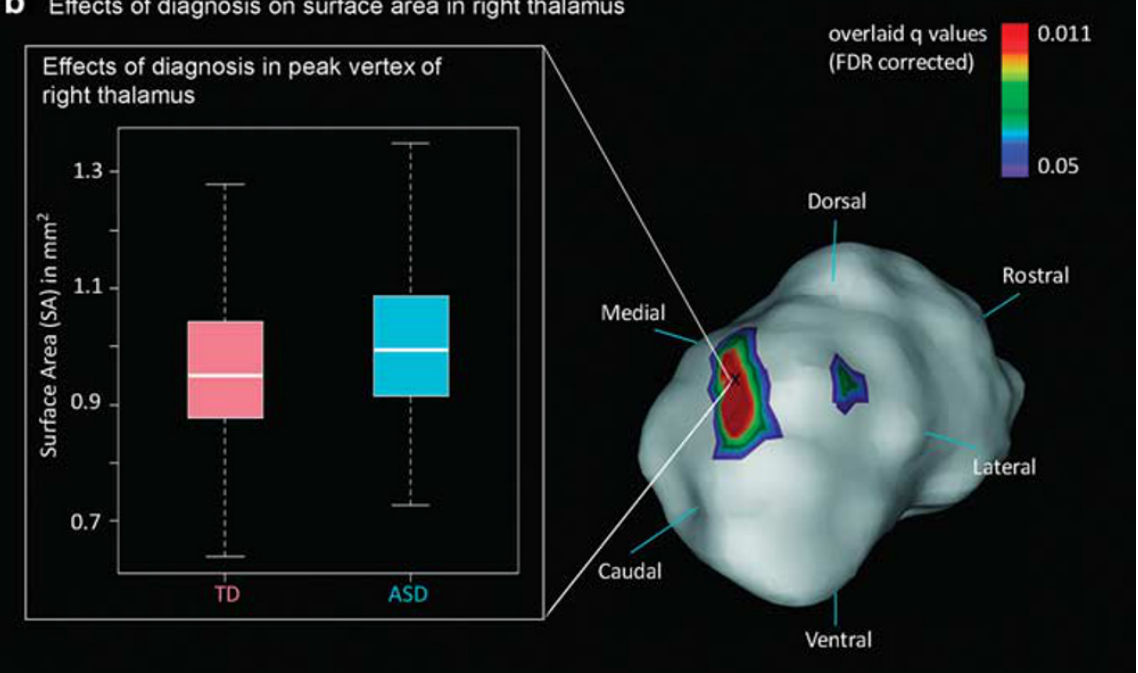

Figure 2 Effects of diagnosis on thalamus surface area. Maps of q-values overlaid on the left and right thalamus. (a) Group differences in surface area were found for three clusters in the left thalamus; within the clusters, the peak vertex is indicated by a black cross. The figure inlay shows the surface area in mm ${ }^{2}$ for the peak vertex of left thalamus. (b) Group differences in surface area were found for two clusters in the right thalamus; within the clusters, the peak vertex is indicated by a black cross. The figure inlay shows the surface area in $\mathrm{mm}^{2}$ for the peak vertex of right thalamus.

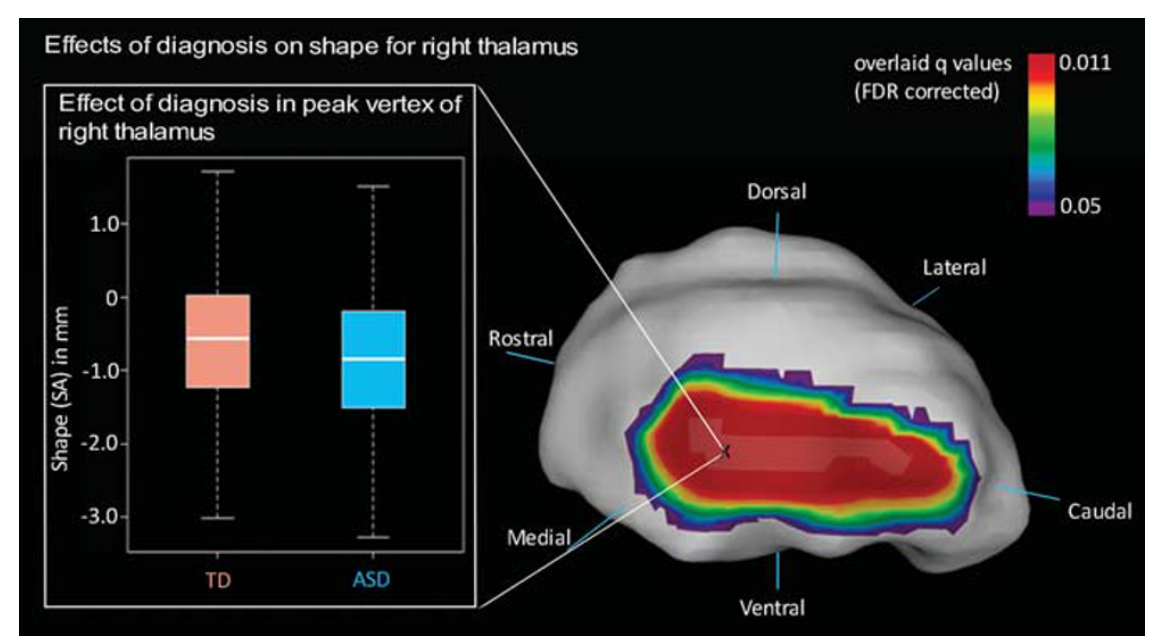

Figure 3 Effects of diagnosis on thalamus shape. Maps of q-values overlaid on right thalamus. Group differences in shape were found for one cluster in right thalamus; within the clusters, the peak vertex is indicated by a black cross. The figure inlay shows the shape displacement for the peak vertex of right thalamus (negative values indicate a more concave shape, and positive values more convex shape). 


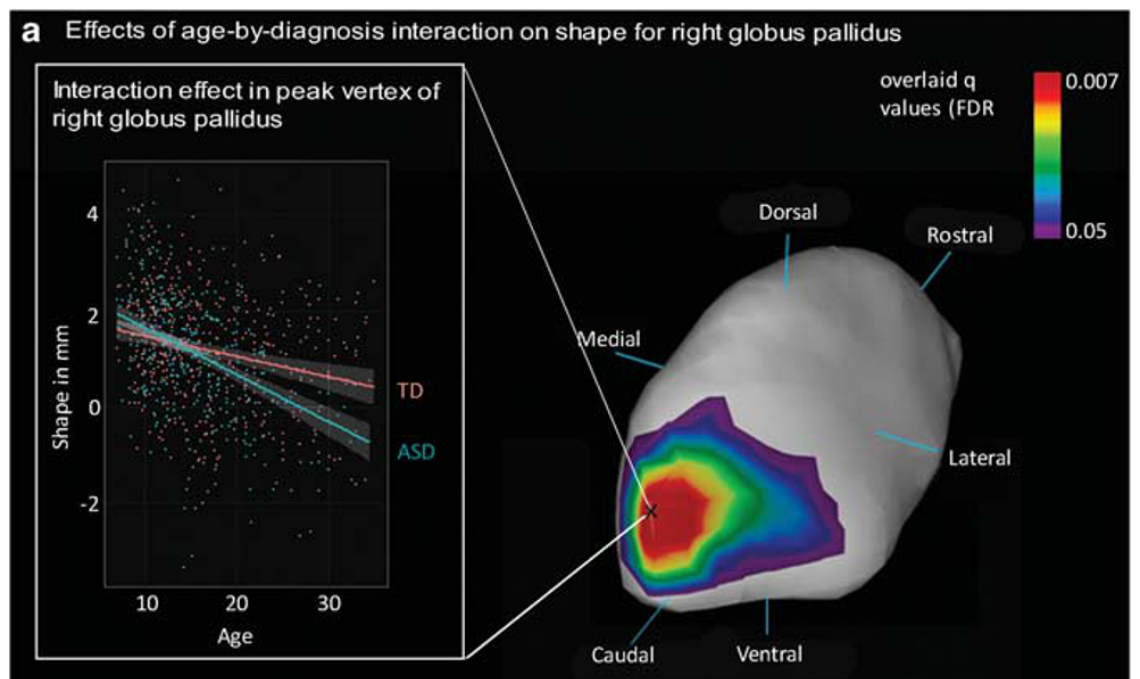

b Effects of age-by-diagnosis interaction on shape for right putamen

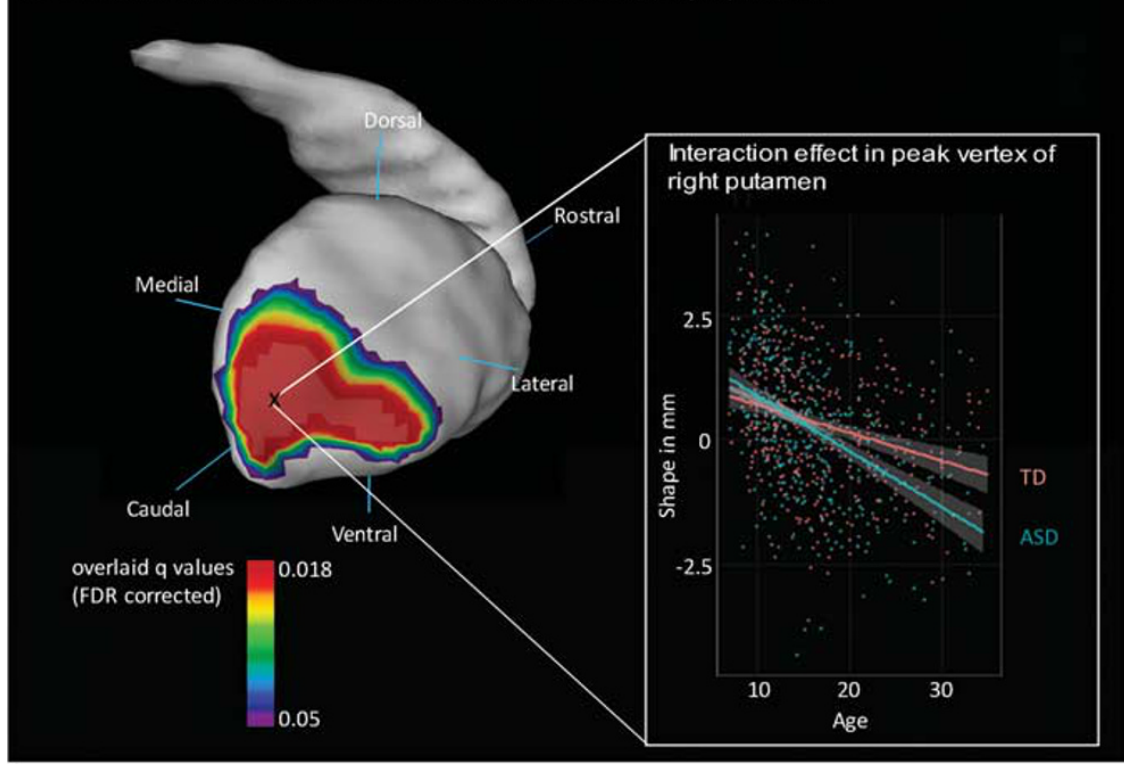

Figure 4 Diagnosis-by-age interaction effects on shape in right pallidum and putamen. Maps of q-values overlaid on right pallidum and putamen. (a) Diagnosis-by-age interaction on shape was found in one cluster in right pallidum; within the clusters, the peak vertex is indicated by a black cross. The figure inlay shows the shape displacement for the peak vertex of right pallidum (negative values indicate a more concave, and positive values more convex shape). (b) Diagnosis-by-age interaction on shape was found in one cluster in right putamen; within the clusters, the peak vertex is indicated by a black cross. The figure inlay shows the shape displacement for the peak vertex of right putamen.

right thalamus. The interaction effects of age-by-diagnosis remained significant for shape of right putamen $(q=0.005)$ and right pallidum $(q=0.003)$. No significant effects or trends were found for volumes in any region after accounting for medication effects.

\section{DISCUSSION}

In the present study, we comprehensively examined volume, surface area, and shape in thalamus, striatum, and pallidum in a large sample of children, adolescents, and young adults with ASD, relative to TD. We found greater surface area bilaterally in the thalami of participants with ASD compared with TD, and more concave shape of the right thalamus in ASD. We also found that shape of right globus pallidus and putamen showed a steeper increase in concavity with age in the ASD group. Finally, we found associations with symptom severity within a thalamic surface area cluster and in the morphology of the globus pallidus. Taken together, these findings suggest that there are subtle differences in subcortical morphology in ASD. Although this study was cross-sectional, our findings also suggest that there may be atypical developmental trajectories in ASD in the shape of pallidum, putamen, and thalamus from childhood to adulthood.

A growing literature on the development of shape and surface area of subcortical structures suggests that this process is both protracted and heterochronous, with regionally specific patterns of expansion and contraction (Raznahan et al, 2014). Thus, while many previous studies of 


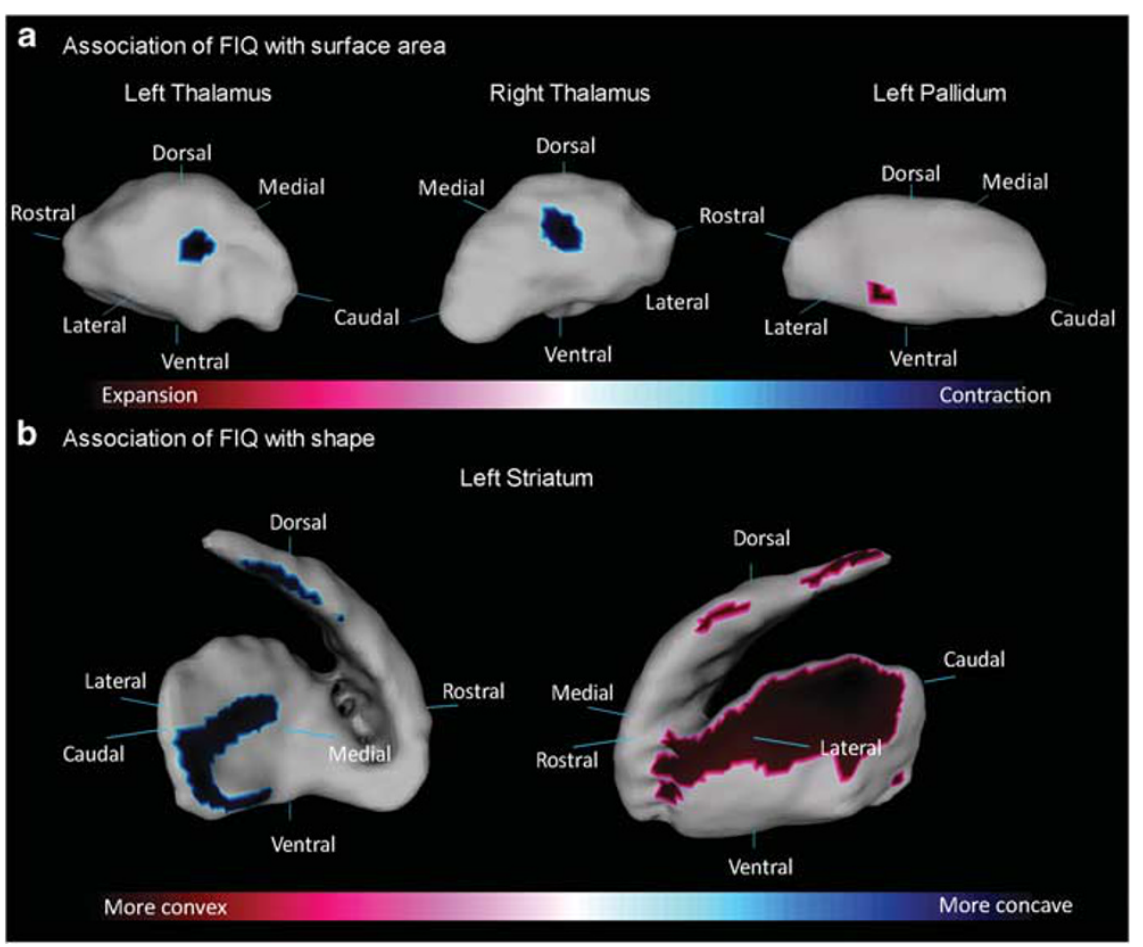

Figure 5 Effects of full-scale IQ (FIQ) on surface area and shape. (a) Thresholded t-values for effects of FIQ on surface area are overlaid on left and right thalamus and left pallidum. (b) Thresholded $t$-values for effects of FIQ on shape are overlaid on left striatum.

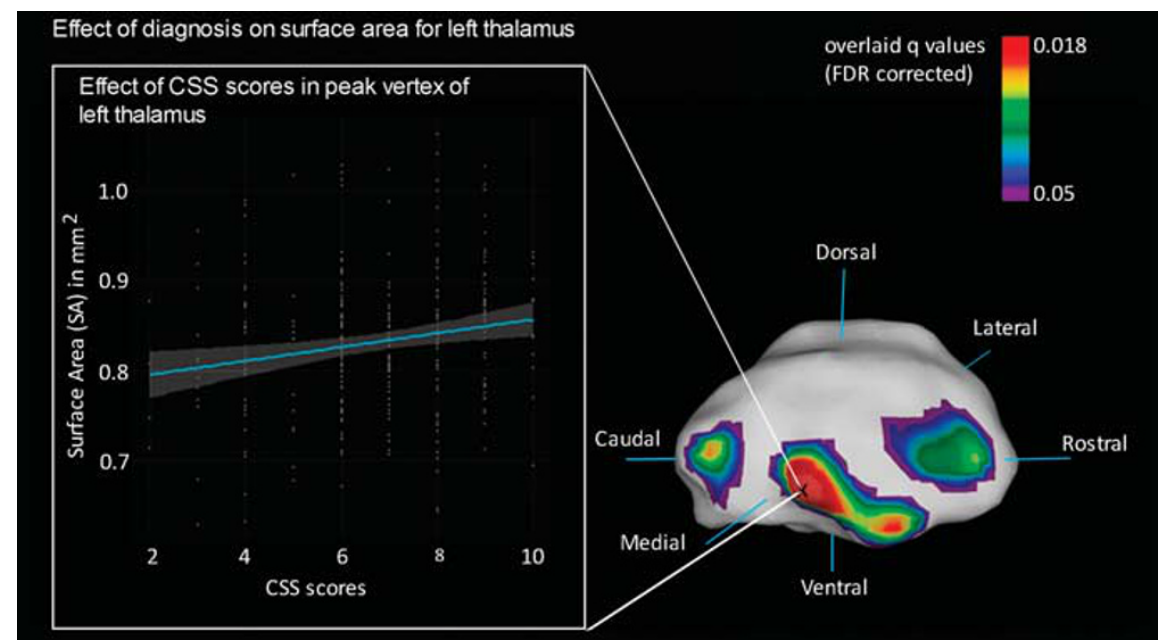

Figure 6 Effects of calibrated symptom severity (CSS) scores on surface area of the left thalamus. Maps of q-values for the main effect of diagnosis (as in Figure 2a) overlaid on the left thalamus. Effects of symptom severity on surface area were found for three clusters in the left thalamus; within the clusters, the peak vertex is indicated by a black cross. The figure inlay shows the surface area for the peak vertex of left thalamus.

subcortical structure in ASD have used volume measures (Giedd et al, 1996; Langen et al, 2007; Lin et al, 2015), these are insensitive to the functional and developmental differentiation within these structures. In contrast, accounting for effects of age, we have shown a specific and persistent increase in surface area in medial and posterior thalamus regions corresponding to the pulvinar and mediodorsal nucleus. Furthermore, we showed that the increase in surface area in left medial thalamus was more pronounced in more severely affected ASD participants compared with less severely affected ASD participants. Although MRI cannot resolve cellular-level differences underlying this expansion, differences in thalamic surface area may be related to differences in myelination or other properties of white matter axons innervating this region (Hirai and Jones, 1989) or to differences in dendritic or neuropil compartments (Fosse et al, 1989). Human and macaque studies indicate prominent connections between the pulvinar nucleus and temporal (Behrens et al, 2003), parieto-occipital, and prefrontal cortices (Arcaro et al, 2015). In ASD, lower connection probability between the posterior thalamus and parietooccipital cortex has been demonstrated (Nair et al, 2013). 


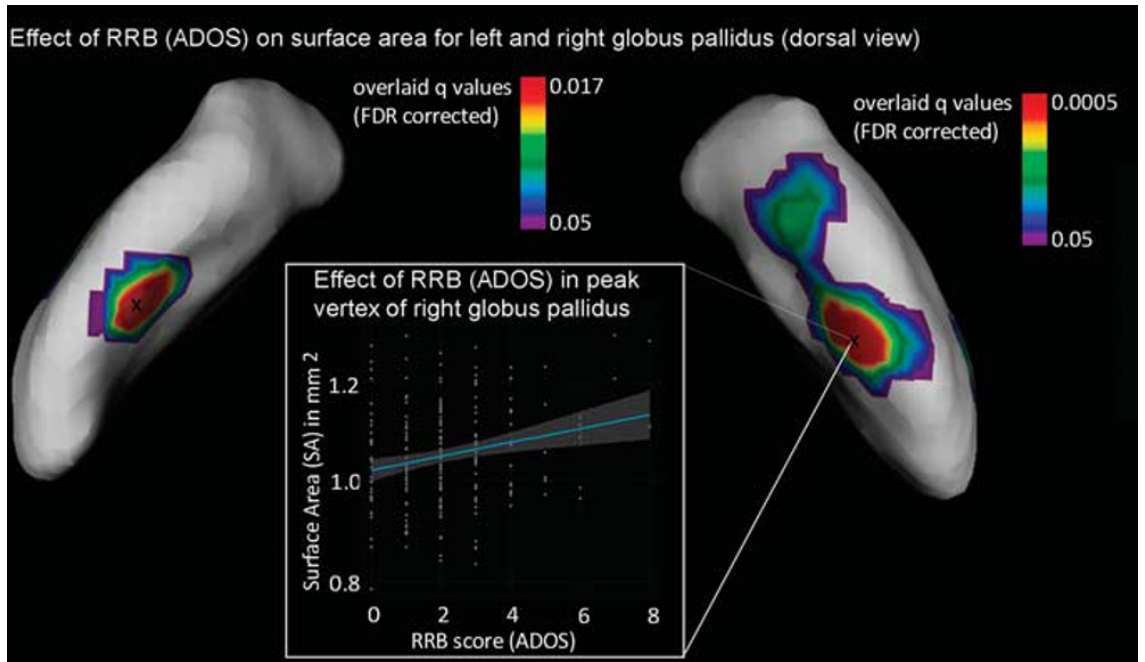

Figure 7 Effects of restricted, repetitive behavior (RRB) scores on surface area for left and right globus pallidus. Maps of q-values overlaid on left and right globus pallidus. Effects of RRB scores as assessed with the Autism Diagnostic Observation Schedule (ADOS) on surface area were found in one cluster in bilateral globus pallidus; within the clusters, the peak vertices are indicated by a black cross. The figure inlay shows the surface area for the peak vertex of right globus pallidus. The relationship between surface area and RRB scores in left globus pallidus followed a similar slope and is not shown here.

Our findings suggest that cellular-level properties within the thalamus may contribute to these differences in connectivity. Functionally, abnormalities in the pulvinar may have particular relevance to well-documented differences in attention skills in ASD (Allen and Courchesne, 2001).

Development of the basal ganglia and thalamus follows known anatomical connectivity patterns (Raznahan et al, 2014), that is, similar rates of maturation are found among interconnected regions. Here we found a steeper increase in concavity in both right caudal putamen and pallidum in ASD, following a known striatopallidal projection (Hazrati and Parent, 1992). Functionally, the caudal pallidum contains both limbic- and sensorimotor-associated zones (Hazrati and Parent, 1992), whereas caudal putamen is principally associated with motor networks (Haber, 2003). A more concave shape of the posterior putamen has previously been linked to decreased motor skills in ASD (Qiu et al, 2010). Our findings together with this previous work suggest a link between morphological alterations in the basal ganglia and motor symptoms in ASD (Jansiewicz et al, 2006; Ming et al, 2007).

Findings on volume measurements of subcortical structures in ASD have been mixed (Giedd et al, 1996; Lin et al, 2015). Notably, studies using larger sample sizes, including this one, have failed to replicate differences in subcortical volumes (Haar et al, 2014; Qiu et al, 2010). Our findings reinforce the importance of assessing sufficiently large samples in heterogeneous populations such as ASD, and suggest that surfacebased analyses may be more sensitive to detect morphological alterations in ASD than overall volume, as they have the ability to capture localized differences in specific nuclei (Chakravarty et al, 2015; Raznahan et al, 2014).

In the typical population, IQ has been associated with total brain volume (McDaniel, 2005) as well as structure of cortical, white matter, and subcortical regions (Grazioplene et al, 2014; Schumann et al, 2007). This is important to consider for any structural study of ASD: while intellectual disability is not among the diagnostic criteria for ASD, it is an extremely common comorbidity (Yeargin-Allsopp et al, 2003). We assessed effects of FIQ in the present study while accounting for ASD diagnosis, and found significant effects of IQ on surface area in bilateral thalamus and left pallidum and on shape in left putamen and dorsal caudate. While our results are discordant with reports of a specific correlation between FIQ and caudate volume (Grazioplene et al, 2014), they do suggest that any findings of morphological differences in pallidum, putamen, and thalamus should be considered relative to intellectual functioning.

Although we did not find a significant association with a measure of global symptom severity, we did find effects related to repetitive and restricted behaviors. These findings are particularly interesting as the basal ganglia are implicated in cognitive and motor control (Gunaydin and Kreitzer, 2015) and have been linked specifically to repetitive and restricted behaviors in ASD (Chukoskie et al, 2013; Qiu et al, 2010). Effects of restricted, repetitive behaviors from the ADOS subscale were in the dorsal medial parts of bilateral globus pallidus. The dorsal globus pallidus receives projections from the cognitive association zones of the striatum (Hazrati and Parent, 1992). Our findings thus primarily suggest abnormalities in the cognitive zones.

While strengths of this study include a large sample size and surface-based analyses of subcortical structures, there are several limitations to note. We used cross-sectional data; therefore, associations with age must be interpreted with caution and confirmed with longitudinal measurements. There are several limitations inherent in the ABIDE data set, including differences in image acquisition protocols, MRI scanners, cognitive assessments, and approaches used to confirm ASD diagnosis and assess symptoms (i.e. not all studies used the ADOS or ADI-R). While we accounted for site as a grouping variable in all analyses, site differences add variability to our measurements. This database also included relatively sparse sampling of females with ASD; therefore, we focused on males and it is unclear whether effects generalize to females.

In summary, the present study investigated effects of ASD diagnosis, and interaction with age, on volume, surface area, and shape of the thalamus and basal ganglia. We found differences in surface area and shape in the posterior and 
medial thalamus, and steeper changes with age in the pallidum and putamen, suggesting differential development in these regions. These focal abnormalities may have a role in the complex symptom profile of ASD, and contribute to the description of an ASD phenotype, which can help to advance development to treatment strategies. A goal for future research will be to understand how precisely these differences relate to ASD symptoms and whether morphological differences relate to differences in subcortical structural and functional connectivity (Nair et al, 2014).

\section{FUNDING AND DISCLOSURE}

The authors declare no conflict of interest.

\section{ACKNOWLEDGMENTS}

We acknowledge all of the research sites who contributed to the ABIDE database for making this resource publicly available. We also acknowledge all of the families who volunteered their time. This work was funded by the Sinneave Family Foundation, the Alberta Children's Hospital Foundation, a University of Calgary seed grant and a SickKids Foundation, and CIHR-IHDCYH New Investigator Award (to SLB). MS was funded by the Alberta Children's Hospital Research Institute for Child and Maternal Health. MMC is receiving salary support from the Fonds de recherches santé Québec and research funding from the Canadian Institutes of Health Research, National Science and Engineering Research Council of Canada, Weston Brain Institute, Michael J Fox Foundation for Parkinson's Research, Alzheimer's Society of Canada, and Brain Canada.

\section{REFERENCES}

Alexander GE, DeLong MR, Strick PL (1986). Parallel organization of functionally segregated circuits linking basal ganglia and cortex. Annu Rev Neurosci 9: 357-381.

Allen G, Courchesne E (2001). Attention function and dysfunction in autism. Front Biosci 6: D105-D119.

American Psychiatric Association. Diagnostic and Statistical Manual of Mental Disorders: DSM-5. American Psychiatric Association: Washington, DC, 2013.

Arcaro MJ, Pinsk MA, Kastner S (2015). The anatomical and functional organization of the human visual pulvinar. $J$ Neurosci 35: 9848-9871.

Ballmaier M, Schlagenhauf F, Toga A, Gallinat J, Koslowski M, Zoli M et al (2008). Regional patterns and clinical correlates of basal ganglia morphology in non-medicated schizophrenia. Schizophr Res 106: 140-147.

Bechara A (2000). Emotion, decision making and the orbitofrontal cortex. Cereb Cortex 10: 295-307.

Behrens TEJ, Johansen-Berg H, Woolrich MW, Smith SM, WheelerKingshott CAM, Boulby PA et al (2003). Non-invasive mapping of connections between human thalamus and cortex using diffusion imaging. Nat Neurosci 6: 750-757.

Belmonte MK, Allen G, Beckel-Mitchener A, Boulanger LM, Carper RA, Webb SJ (2004). Autism and abnormal development of brain connectivity. J Neurosci 24: 9228-9231.

Bonelli RM, Cummings JL (2007). Frontal-subcortical circuitry and behavior. Dialog Clin Neurosci 9: 141-151.

Borghammer P, Østergaard K, Cumming P, Gjedde A, Rodell A, Hall N et al (2010). A deformation-based morphometry study of patients with early-stage Parkinson's disease. Eur J Neurol 17: 314-320.
Campbell DB, D’Oronzio R, Garbett K, Ebert PJ, Mirnics K, Levitt P et al (2007). Disruption of cerebral cortex MET signaling in autism spectrum disorder. Ann Neurol 62: 243-250.

CDC (2014). CDC estimates 1 in 68 children has been identified with autism spectrum disorder. Centers Disease Control Prevention. Available at: http://www.cdc.gov/media/releases/2014/p0327-aut ism-spectrum-disorder.html (last date accessed 28 January 2016).

Chakravarty MM, Bertrand G, Hodge CP, Sadikot AF, Collins DL (2006). The creation of a brain atlas for image guided neurosurgery using serial histological data. Neuroimage 30: 359-376.

Chakravarty MM, Rapoport JL, Giedd JN, Raznahan A, Shaw P, Collins DL et al (2015). Striatal shape abnormalities as novel neurodevelopmental endophenotypes in schizophrenia: a longitudinal study. Hum Brain Mapp 36: 1458-1469.

Chakravarty MM, Steadman P, Eede MC, van Calcott RD, Gu V, Shaw $P$ et al (2013). Performing label-fusion-based segmentation using multiple automatically generated templates. Hum Brain Mapp 34: 2635-2654.

Chukoskie L, Townsend J, Westerfield M (2013). Motor skill in autism spectrum disorders: a subcortical view. Int Rev Neurobiol 113: 207-249.

Collins DL, Pruessner JC (2010). Towards accurate, automatic segmentation of the hippocampus and amygdala from MRI by augmenting ANIMAL with a template library and label fusion. Neuroimage 52: 1355-1366.

Di Martino A, Yan C-G, Li Q, Denio E, Castellanos FX, Alaerts K et al (2014). The autism brain imaging data exchange: towards a large-scale evaluation of the intrinsic brain architecture in autism. Mol Psychiatry 19: 659-667.

Ellegood J, Anagnostou E, Babineau BA, Crawley JN, Lin L, Genestine $M$ et al (2015). Clustering autism: using neuroanatomical differences in 26 mouse models to gain insight into the heterogeneity. Mol Psychiatry 20: 118-125.

Eskildsen SF, Coupé P, Fonov V, Manjón JV, Leung KK, Guizard N et al (2012). BEaST: brain extraction based on nonlocal segmentation technique. Neuroimage 59: 2362-2373.

Fosse VM, Heggelund P, Fonnum F (1989). Postnatal development of glutamatergic, GABAergic, and cholinergic neurotransmitter phenotypes in the visual cortex, lateral geniculate nucleus, pulvinar, and superior colliculus in cats. J Neurosci 9: 426-435.

Frey S, Pandya DN, Chakravarty MM, Bailey L, Petrides M, Collins DL (2011). An MRI based average macaque monkey stereotaxic atlas and space (MNI monkey space). Neuroimage 55: 1435-1442.

Fuccillo MV (2016). Striatal circuits as a common node for autism pathophysiology. Front Neurosci 10: 27.

Genovese CR, Lazar NA, Nichols T (2002). Thresholding of statistical maps in functional neuroimaging using the false discovery rate. Neuroimage 15: 870-878.

Geurts HM, Corbett B, Solomon M (2009). The paradox of cognitive flexibility in autism. Trends Cogn Sci 13: 74-82.

Giedd JN, Snell JW, Lange N, Rajapakse JC, Casey BJ, Kozuch PL et al (1996). Quantitative magnetic resonance imaging of human brain development: ages 4-18. Cereb Cortex 6: 551-560.

Gotham K, Pickles A, Lord C (2009). Standardizing ADOS scores for a measure of severity in autism spectrum disorders. J Autism Dev Disord 39: 693-705.

Graybiel AM (2008). Habits, rituals, and the evaluative brain. Annu Rev Neurosci 31: 359-387.

Grazioplene RG, Ryman GS, Gray JR, Rustichini A, Jung RE, DeYoung CG (2014). Subcortical intelligence: caudate volume predicts IQ in healthy adults. Hum Brain Mapp 36: 1407-1416.

Gunaydin LA, Kreitzer AC (2015). Cortico-basal ganglia circuit function in psychiatric disease. Annu Rev Physiol 78: 327-350.

Haar S, Berman S, Behrmann M, Dinstein I (2014). Anatomical abnormalities in autism? Cereb Cortex 26: 1440-1452.

Haber SN (2003). The primate basal ganglia: parallel and integrative networks. J Chem Neuroanat 26: 317-330. 
Hazrati LN, Parent A (1992). The striatopallidal projection displays a high degree of anatomical specificity in the primate. Brain Res 592: 213-227.

Hillis AE (2014). Inability to empathize: brain lesions that disrupt sharing and understanding another's emotions. Brain 137: 981-997.

Hirai T, Jones EG (1989). A new parcellation of the human thalamus on the basis of histochemical staining. Brain Res Brain Res Rev 14: 1-34.

Ho B-C, Andreasen NC, Ziebell S, Pierson R, Magnotta V (2011). Long-term antipsychotic treatment and brain volumes: a longitudinal study of first-episode schizophrenia. Arch Gen Psychiatry 68: $128-137$.

Hollander E, Anagnostou E, Chaplin W, Esposito K, Haznedar MM, Licalzi E et al (2005). Striatal volume on magnetic resonance imaging and repetitive behaviors in autism. Biol Psychiatry 58: 226-232.

Holmes CJ, Hoge R, Collins L, Woods R, Toga AW, Evans AC (1998). Enhancement of MR images using registration for signal averaging. J Comput Assist Tomogr 22: 324-333.

Hus V, Lord C (2014). The autism diagnostic observation schedule, module 4: revised algorithm and standardized severity scores. J Autism Dev Disord 44: 1996-2012.

Jansiewicz EM, Goldberg MC, Newschaffer CJ, Denckla MB, Landa R, Mostofsky SH (2006). Motor signs distinguish children with high functioning autism and asperger's syndrome from controls. J Autism Dev Disord 36: 613-621.

Kohls G, Schulte-Rüther M, Nehrkorn B, Müller K, Fink GR, Kamp-Becker I et al (2013). Reward system dysfunction in autism spectrum disorders. Soc Cogn Affect Neurosci 8: 565-572.

Krumm N, O’Roak BJ, Shendure J, Eichler EE (2014). A de novo convergence of autism genetics and molecular neuroscience. Trends Neurosci 37: 95-105.

Langen M, Bos D, Noordermeer SDS, Nederveen H, Engeland H, Van, Durston S (2014). Changes in the development of striatum are involved in repetitive behavior in autism. Biol Psychiatry 76: 405-411.

Langen M, Durston S, Staal WG, Palmen SJMC, vanEngeland H (2007). Caudate nucleus is enlarged in high-functioning medication-naive subjects with autism. Biol Psychiatry 62: 262-266.

Lerch JP, Carroll JB, Spring S, Bertram LN, Schwab C, Hayden MR et al (2008). Automated deformation analysis in the YAC128 Huntington disease mouse model. Neuroimage 39: 32-39.

Lin H-Y, Ni H-C, Lai M-C, Tseng W-YI, Gau SS-F (2015). Regional brain volume differences between males with and without autism spectrum disorder are highly age-dependent. Mol Autism 6: 29.

Lord C, Rutter M, DiLavore P, Risi S. Autism Diagnostic Observation Schedule-WPS Edition. Western Psychological Services: Los Angeles, CA, 1999.

Lorensen WE, Cline HE (1987). Marching cubes: a high resolution 3D surface construction algorithm. ACM Siggraph Comput Graph 21: $163-169$

McAlonan GM, Suckling J, Wong N, Cheung V, Lienenkaemper N, Cheung $\mathrm{C}$ et al (2008). Distinct patterns of grey matter abnormality in high-functioning autism and Asperger's syndrome. J Child Psychol Psychiatry 49: 1287-1295.

McDaniel M (2005). Big-brained people are smarter: a metaanalysis of the relationship between in vivo brain volume and intelligence. Intelligence 33: 337-346.

McFadden K, Minshew NJ (2013). Evidence for dysregulation of axonal growth and guidance in the etiology of ASD. Front Hum Neurosci 7: 671.
Ming X, Brimacombe M, Wagner GC (2007). Prevalence of motor impairment in autism spectrum disorders. Brain Dev 29: 565-570.

Nair A, Keown CL, Datko M, Shih P, Keehn B, Müller RA (2014). Impact of methodological variables on functional connectivity findings in autism spectrum disorders. Hum Brain Mapp 35: 4035-4048.

Nair A, Treiber JM, Shukla DK, Shih P, Müller R-A (2013). Impaired thalamocortical connectivity in autism spectrum disorder: a study of functional and anatomical connectivity. Brain 136: 1942-1955.

Navari S, Dazzan P (2009). Do antipsychotic drugs affect brain structure? A systematic and critical review of MRI findings. Psychol Med 39: 1763-1777.

Nomi JS, Uddin LQ (2015). Face processing in autism spectrum disorders: from brain regions to brain networks. Neuropsychologia 71: 201-216.

O’Roak BJ, Vives L, Girirajan S, Karakoc E, Krumm N, Coe BP et al (2012). Sporadic autism exomes reveal a highly interconnected protein network of de novo mutations. Nature 485: 246-250.

Peñagarikano O, Abrahams BS, Herman EI, Winden KD, Gdalyahu A, Dong $\mathrm{H}$ et al (2011). Absence of CNTNAP2 leads to epilepsy, neuronal migration abnormalities, and core autism-related deficits. Cell 147: 235-246.

Qiu A, Adler M, Crocetti D, Miller MI, Mostofsky SH (2010). Basal ganglia shapes predict social, communication, and motor dysfunctions in boys with autism spectrum disorder. J Am Acad Child Adolesc Psychiatry 49: 539-551, 551.e1-551.e4.

Raznahan A, Shaw PW, Lerch JP, Clasen LS, Greenstein D, Berman R et al (2014). Longitudinal four-dimensional mapping of subcortical anatomy in human development. Proc Natl Acad Sci USA 111: 1592-1597.

Rutter M, Couteur A, Le, Lord C. Autism Diagnostic InterviewRevised. Western Psychological Services: Los Angeles, CA, USA.

Schumann CM, Hamstra J, Goodlin-Jones BL, Kwon H, Reiss AL, Amaral DG (2007). Hippocampal size positively correlates with verbal IQ in male children. Hippocampus 17: 486-493.

Shaw P, De Rossi P, Watson B, Wharton A, Greenstein D, Raznahan A et al (2014). Mapping the development of the basal ganglia in children with attention-deficit/hyperactivity disorder. J Am Acad Child Adolesc Psychiatry 53: 780-789, e11.

Smith JM, Xu J, Powell EM (2012). Age dependent forebrain structural changes in mice deficient in the autism associated gene Met tyrosine kinase. NeuroImage Clin 1: 66-74.

Wei H, Alberts I, Li X (2014). The apoptotic perspective of autism. Int J Dev Neurosci 36: 13-18.

Yeargin-Allsopp M, Rice C, Karapurkar T, Doernberg N, Boyle C, Murphy C (2003). Prevalence of autism in a US metropolitan area. JAMA 289: 49-55.

(c) (1) (-) $\Theta$ This work is licensed under a Creative Commons Attribution-NonCommercial-NoDerivs $\quad 4.0$ International License. The images or other third party material in this article are included in the article's Creative Commons license, unless indicated otherwise in the credit line; if the material is not included under the Creative Commons license, users will need to obtain permission from the license holder to reproduce the material. To view a copy of this license, visit http://creativecommons.org/licenses/by-nc-nd/4.0/

Supplementary Information accompanies the paper on the Neuropsychopharmacology website (http://www.nature.com/npp) 